PACS: 78.60.Fi; 71.55.Gs; 72.10.-d; 71.70.Gm; 78.20.Ls

\title{
Momentum relaxation of hot electrons during radiative intraband indirect transitions in $\mathrm{ZnS}: \mathrm{Cr}$
}

\author{
N.A. Vlasenko, A.E. Belyaev, Z.L. Denisova, Ya.F. Kononets, A.V. Komarov, and L.I. Veligura \\ V. Lashkaryov Institute of Semiconductor Physics, NAS of Ukraine, 41, prospect Nauky, 03028 Kyiv, Ukraine
}

\begin{abstract}
A structureless emission in the visible region was revealed in $\mathrm{ZnS}$ : $\mathrm{Cr}$ thin-film electroluminescent structures (TFELS) apart the main near-infrared emission resulted from the ${ }^{5} \mathrm{E} \rightarrow{ }^{5} \mathrm{~T}_{2}$ transition in the $3 \mathrm{~d}$ shell of the $\mathrm{Cr}^{2+}$ ion. This emission stems from intraband indirect transitions of hot electrons. The comparison between experimental intensity spectral dependence and the same dependence calculated for different mechanisms of the momentum relaxation shows that the scattering on charged impurities takes place during this emission. The $\mathrm{Cr}^{+}$ions existing in $\mathrm{ZnS}: \mathrm{Cr}$ in addition to predominant $\mathrm{Cr}^{2+}$ ions serve as scattering charge centers. This is confirmed by study of the magnetic field $(H)$ effect on the intensity $(I)$ of the hot electron emission at $4.2 \mathrm{~K}$. The experimental dependence $I(H)$ coincides with the calculated magnetic field dependence of the exchange scattering cross-section of $\mathrm{Cr}^{+}$ion with the spin 5/2 and $g$ factor 2. This result also indicates that both the Coulomb interaction with $\mathrm{Cr}^{+}$ions and the exchange scattering on them present during the hot electron emission in the $\mathrm{ZnS}: \mathrm{Cr}$ TFELS.
\end{abstract}

Keywords: hot electrons, electroluminescence, thin films, $\mathrm{ZnS}: \mathrm{Cr}$, momentum relaxation, charge impurities, exchange scattering, $\mathrm{Cr}^{+}$ions.

Manuscript received 13.12.04; accepted for publication 18.05.05.

\section{Introduction}

The following radiative transitions of hot electrons generated by high energy photons or by high electric field are feasible in semiconductors: interband transitions, "band-impurity" transitions, and intraband transitions. The latter may be transitions between different valleys of the conduction band and indirect intraband transitions within a single valley of this band. In virtue of the law of momentum conservation, the intraband indirect transitions in both emission and absorption of free electrons are possible only if there is a mechanism of the scattering of their kinetic energy. The main mechanism of scattering is interaction with phonons (optical and acoustic) and with charge impurities or lattice defects $[1,2]$. In the presence of paramagnetic centers, the exchange mechanism that includes the spin-flip scattering is also possible [3]. Radiative intraband transitions have been observed in reverse biased diodes of II-VI, III-V compounds, $\mathrm{Si}$, Ge, and $\mathrm{SiC}[2,4]$. In particular, such transitions have been studied in ZnS Shottky diodes [2, 4] and in ZnS thinfilm electroluminescent structures (TFELS) excited by high alternating electric fields [5].

The hot electron emission resulted from intraband indirect transitions should inherently represent a wide structureless band limited in the short wavelength region by the electron maximum energy. This was confirmed by a simplified theoretical model of such an emission developed in [6]. The model is based on the consideration of the hot electron emission as a process opposite to the hot electron absorption with allowance for the detailed balance principle. The basic parameter that determines the spectral distribution of the emission is the absorption cross-section $\left(\sigma_{\mathrm{abs}}\right)$. The latter depends on the photon energy $(h v)$ for the different scattering mechanisms as follows $[7,8]$ :

$\sigma_{\text {abs }}=\sigma_{0}(h v)^{-r}\left(E_{k} / h v-1\right)^{1 / 2}\left(2 E_{g} / h v-1\right)$,

where $\sigma_{0}$ is a value independent of $h v ; E_{k}$ is the energy of a level from which the transition occurs; $r$ is equal $3 / 2,5 / 2$, or $7 / 2$ for the scattering by acoustic phonons, optic phonons, and charge impurities, respectively.

It has been shown by comparison of experimental and calculated data for some semiconductors ( $\mathrm{ZnS}$, CdTe, etc.) [2] that this model gives an adequate account for spectral distribution and other characteristics of the intraband hot electron emission observed. At the same time, a marked discrepancy between the experimental and calculated spectral dependences of the emission quantum output has been revealed near the fundamental absorption edge. This discrepancy is explained, firstly, by self-absorption of the emission and, secondly, by an infringement of a model assumption concerning the parabolicity of the conduction band.

The influence of the exchange scattering on the hot electron emission has not been studied up to now in spite of its known essential effects in optical, electrical and 


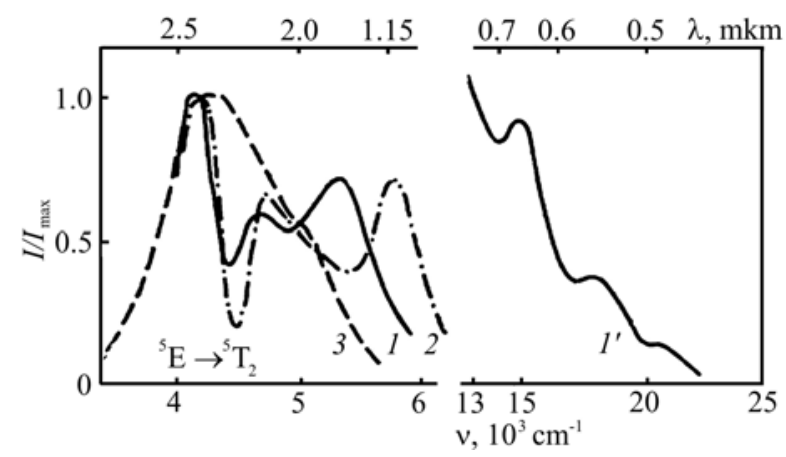

Fig. 1. Electroluminescence spectrum of $\mathrm{ZnS}: \mathrm{Cr}\left(1,1^{\prime}\right)$ and $\mathrm{ZnSe}: \mathrm{Cr}(2) \mathrm{TFELS}$ as well as photoluminescence spectrum of $\mathrm{ZnSe}: \mathrm{Cr}$ crystal (3).

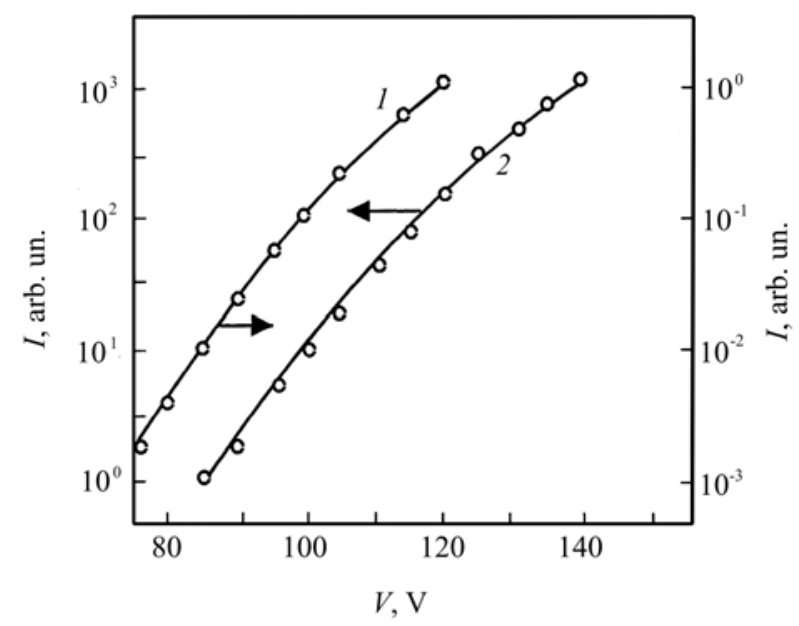

Fig. 2. Voltage dependence of integrated intensity of visible (1) and NIR (2) emission of ZnS:Cr TFELS. $f=5 \mathrm{kHz}$.

magnetic properties of semiconductors (see, e.g. [3, 9, $10])$. The authors of given papers have revealed [11] in $\mathrm{ZnS}(\mathrm{Se})$ :Cr TFELS not only the emission in the nearinfrared region $(1.8 \ldots 2.7 \mu \mathrm{m})$ that results from ${ }^{5} \mathrm{E} \rightarrow{ }^{5} \mathrm{~T}_{2}$ transition in the $3 \mathrm{~d}$ shell of $\mathrm{Cr}^{2+}$ ion, but also the weaker emission ranged from 0.4 to $1.0 \mu \mathrm{m}$ with the intensity increasing monotonically when $h v$ decreases apart its modulation by interference extrema (Fig. 1). This emission was assumed to be due to intraband transitions of hot electrons. The purpose of this paper is to confirm this assumption and to ascertain the mechanism of the momentum relaxation of high-energy electrons in the presence of the high concentration of paramagnetic centers in $\mathrm{ZnS}: \mathrm{Cr}$. The influence of the chromium concentration and the applied voltage magnitude on the spectral distributions of intensity $(I)$ of the hot electron emission was studied. The comparison of the experimental dependence $I$ versus $h v$ and this dependence calculated according to the above mentioned model for different scattering mechanism has been done. In addition, the dependence of $I$ on the magnetic field at $4.2 \mathrm{~K}$ was carried out to ascertain the contribution of exchange scattering to the momentum relaxation of hot electrons and to establish the charge of $\mathrm{Cr}$ ions responsible for such a scattering.

\section{Experimental procedures}

The samples of TFELS under study consisted of the following layers successively deposited on a glass substrate: a transparent ITO electrode, an electroluminescent (EL) $\mathrm{ZnS}: \mathrm{Cr}$ film with the thickness of $\sim 0.8 \mu \mathrm{m}$, a $50-\mathrm{nm}$ thick semi-insulating $\mathrm{SiO}_{x}$ film with $x \sim 1.2$, and an upper Al electrode. The EL film was deposited by electron-beam evaporation and was annealed after that in vacuum at $\sim 500{ }^{\circ} \mathrm{C}$. Doping the EL film was performed by simultaneous evaporation of $\mathrm{ZnS}$ and $\mathrm{Cr}$ from separate sources. The chromium concentration varied from 0.2 to $1.0 \mathrm{wt} \%$.

Electroluminescence was excited by sinusoidal voltage $(V)$ of $5 \mathrm{kHz}$ frequency. The voltage dependence of the integrated intensity of the emission in the visible region and in the near-infrared (NIR) region was measured by a photomultiplier and a $\mathrm{PbS}$ detector, respectively, using the corresponding filters. The spectral dependence of the visible emission intensity was recorded with a KSVU-23 spectrometer.

Analysis of the photodepolarization (PDP) spectrum of the ZnS:Cr TFELS yields an information about defects appearing in the $\mathrm{ZnS}$ film after its doping with $\mathrm{Cr}$. This spectrum represents the spectral dependence of the photocurrent generated by testing monochromatic light in a previously charged sample under the action of a residual polarization field. The PDP spectrum was measured using the method described in [12]. The sample was charged by application of a short duration $\mathrm{dc}$ voltage equal to $\sim V_{\text {th }} / 2$, where $V_{\text {th }}$ is the threshold voltage of the electroluminescence. Illumination of the sample during the PDP spectrum recording was performed from the ITO side. Interference extrema in this spectrum were averaged. The spectrum was not corrected by the equal number of testing photons.

The influence of the magnetic field $(H)$ on the integrated intensity of the hot electron emission was studied at the temperature of $4.2 \mathrm{~K}$ and $H$ varied up to 3.5T. The magnetic field was directed in parallel to the electric field in the $\mathrm{ZnS}: \mathrm{Cr}$ film. The emission was taken out from a cryostat through a light guide.

\section{Results and discussion}

Electroluminescence in both the visible and NIR regions appeared at the mean field strength in the $\mathrm{ZnS}: \mathrm{Cr}$ film over $1 \mathrm{MV} / \mathrm{cm}$. It has been found that the integrated emission intensity in both regions shows the same exponential dependence on the applied voltage (Fig. 2). Such a dependence is typical for the impact excitation mechanism of electroluminescence that takes place in TFELS doped with Mn, rare-earth ions and other impurities [13]. Therefore, the identical voltage 


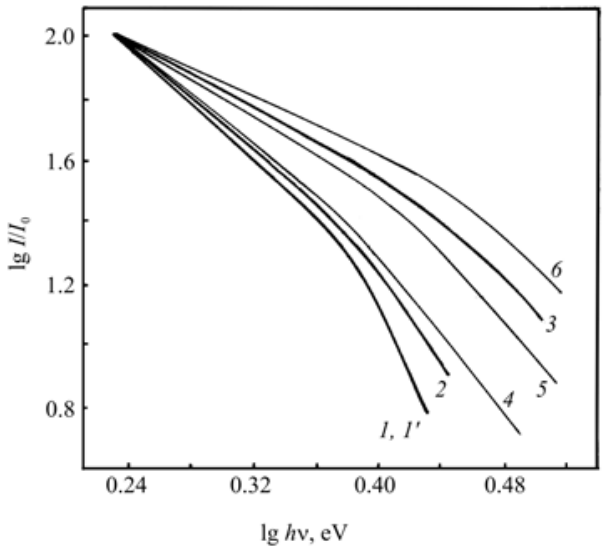

Fig. 3. Spectral dependence of intensity of hot electron emission of $\mathrm{ZnS}: \mathrm{Cr}$ TFELS (1) and $\mathrm{ZnS}$ diodes (2), experimental (3) and calculated in the case of different scattering mechanisms on charge impurities (4), on optic and acoustic phonons $(5,6$, respectively). Temperature, $\mathrm{K}: 300$ ( 1 $6)$ and $4.2\left(1^{\prime}\right) . C_{\mathrm{Cr}}, \mathrm{wt} . \%: 1.0\left(1,1^{\prime}\right)$ and $0.5(2)$.

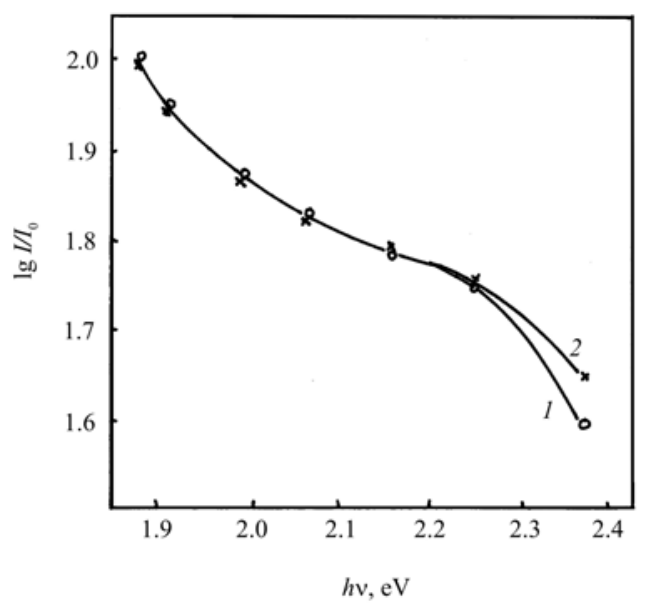

Fig. 4. Spectral dependence of intensity of hot electrons of ZnS:Cr TFELS at various applied voltages: $V=137 \mathrm{eV}(1)$ and 150 (2). $C_{\mathrm{Cr}}=0.5 \mathrm{wt} \%$.

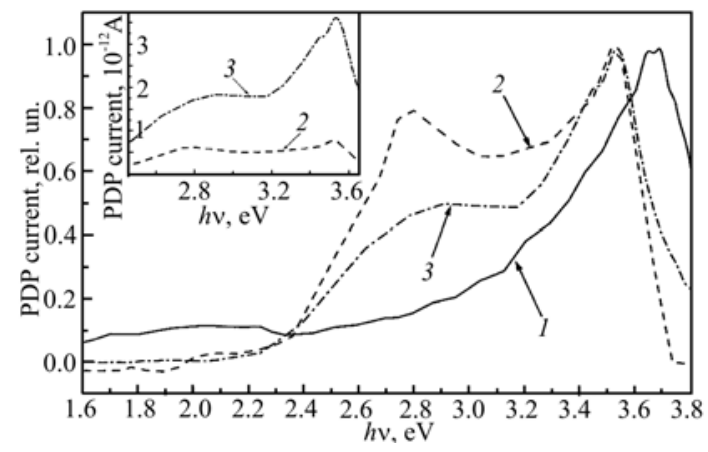

Fig. 5. Normalized PDP spectrum of $\mathrm{ZnS}$ (1), $\mathrm{ZnS}: \mathrm{Cr}$, 0.2 wt.\% (2) and ZnS:Cr, 0.5 wt.\% (3) TFELS. Insert: absolute magnitude of PDP current as a function of photon energy for the same $\mathrm{ZnS}$ :Cr TFELS. dependence of the $\mathrm{Cr}^{2+}$ ion NIR emission and the visible emission indicate that free electrons accelerated up to the high energy by the strong electric field take part in both processes. While the low-intensity and structureless spectral dependence of the emission in the visible region (Fig. 1) evidences that this emission results from intraband indirect transitions.

The spectral dependence of the intensity of the hot electron emission for two samples with different $\mathrm{Cr}$ concentration is shown in Fig. 3 (curves 1 and 2). The dependence becomes steeper, in particular at $h v>2.3 \mathrm{eV}$, when $C_{\mathrm{Cr}}$ increases. The shape of this dependence does not change with applied voltage increasing except for its high-energy section that intensifies in this case (Fig. 4).

The data for $\mathrm{ZnS}$ diodes taken from the paper [2] are also given in Fig. 3: the experimental spectral dependence of the quantum output of the hot electron emission (curve 3 ) is shown together with dependences calculated according to the above mentioned model for the various scattering mechanisms (curves $4-6$ ). It is seen that experimental data for undoped $\mathrm{ZnS}$ are very close to dependences calculated for the phonon scattering mechanism. However, in the $\mathrm{ZnS}$ :Cr TFELS the spectral dependence of the emission intensity is significantly steeper and similar in its low-energy section to the dependence calculated for scattering by charged impurities.

Some discrepancy between the experimental and calculated dependences observed at $h v>2.3 \mathrm{eV}$ becomes considerable when the $\mathrm{Cr}$ concentration is higher. This discrepancy may be attributed to the absorption of photons by defects, which appears in $\mathrm{ZnS}$ after doping with $\mathrm{Cr}$. Indeed, in the PDP spectrum of the $\mathrm{ZnS}: \mathrm{Cr}$ TFELS there are two broad intensive bands of the impurity photocurrent with the maxima at $\sim 2.8$ and $\sim 3.5 \mathrm{eV}$ (Fig. 5). The peak of the inherent photocurrent in the PDP spectrum of undoped $\mathrm{ZnS}$ corresponds to the bandgap energy $\left(E_{g}=3.68 \mathrm{eV}\right)$. Two bands revealed in the PDP spectrum for the first time coincide as to their spectral position with two regions $(2.3<h v<3.0 \mathrm{eV}$ and $3.0<h v<3.6 \mathrm{eV}$ ) in the spectrum of photo-recharging of $\mathrm{Cr}^{2+}$ ions into $\mathrm{Cr}^{+}$ions, which have been found out using electron paramagnetic resonance (EPR) experiments (see [14] and references therein). The former was explained before as the transition of electrons from the valence band directly into $3 \mathrm{~d}$ shell of $\mathrm{Cr}^{2+}$ ions, and the latter was attributed to band-to-band transitions. However, such an interpretation contradicts with existence of the photocurrent in the first region and with $h v<E_{g}$ within the second region. A new interpretation of both regions observed in the PDP spectrum and in the $\mathrm{Cr}^{2+} \rightarrow \mathrm{Cr}^{+}$photo-recharging spectrum in $\mathrm{ZnS}: \mathrm{Cr}$ has been given in [15]. The higherenergy region was assumed to be related to the photoionization of the deep isovalent trap $\left[\mathrm{Cr}_{\mathrm{Zn}}^{2+}\right]^{0}$ that appears when $\mathrm{Zn}^{2+}$ ion is substituted by $\mathrm{Cr}^{2+}$ ion. It has 
been shown in [16] that analogous defect arises in $\mathrm{ZnS}: \mathrm{Mn}$ as a consequence of the substitution of $\mathrm{Mn}^{2+}$ ion for $\mathrm{Zn}^{2+}$ ion. As to the second region, in which the energy levels of all deep acceptors known in $\mathrm{ZnS}$ $\left(\left[\mathrm{Cu}_{\mathrm{Zn}}^{+}\right]^{-},\left[\mathrm{Ag}_{\mathrm{Zn}}^{+}\right]^{-}, \mathrm{V}_{\mathrm{Zn}}^{2-}\right.$, etc. $)$ are located, the photoionization of $\left[\mathrm{Cr}_{\mathrm{Zn}}^{+}\right]^{-}$acceptors was assumed to be responsible for it. It is known [14] that there is a number of $\mathrm{Cr}^{+}$ions in $\mathrm{ZnS}: \mathrm{Cr}$ in addition to the predominant double charged chromium ions.

The assumption about the presence of some number of $\mathrm{Cr}^{+}$ions in the $\mathrm{ZnS}: \mathrm{Cr}$ films turned out to be necessary for the explanation of the momentum relaxation mechanism during radiative intraband indirect transitions of hot electrons. It follows from the spectral dependence of the intensity of the hot electron emission that the main scattering mechanism in the $\mathrm{ZnS}: \mathrm{Cr}$ TFELS is the interaction with charged impurities. Intrinsic charged defects are not able to give the predominance of this mechanism due to their rather small concentration $\left(<10^{17} \mathrm{~cm}^{-3}\right)$ in both the undoped $\mathrm{ZnS}$ and the $\mathrm{ZnS}$ doped with an isovalent impurity. A confirmation of this is the identical spectral dependence of the intensity of the hot electron emission at 300 and 4.2 K (see curves 1 and 1' in Fig. 3), whereas the number of charged defects must significantly decrease at low temperature. $\mathrm{Cr}^{2+}$ ions form the neutral centers which cannot result in considerable bending the electron trajectory. There is nothing to do but to assume the existence of $\mathrm{Cr}^{+}$ions in the $\mathrm{ZnS}: \mathrm{Cr}$ films as the scattering charge impurities.

If this assumption is true, the marked exchange scattering aside the Coulomb interaction should be expected at low temperatures because of $3 \mathrm{~d}^{5}$ electron configuration of $\mathrm{Cr}^{+}$ion. Therefore, the influence of the magnetic field on $I$ should be observed at these temperatures. In the magnetic field, the spin polarization

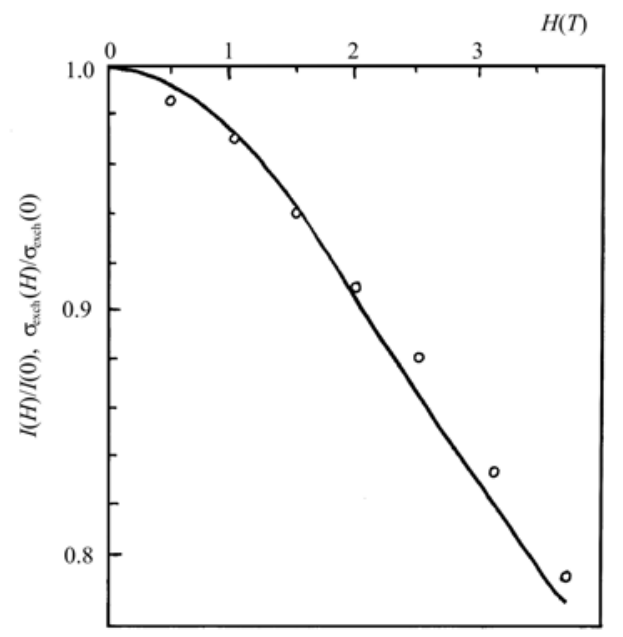

Fig. 6. Magnetic-field dependencies of intensity of hot electron of $\mathrm{ZnS}: \mathrm{Cr}$ TFELS (points) and of exchange scattering cross-section calculated by relations (2) for scattering centers with $s=5 / 2$ and $g=2$ (solid curve) $T=4.2 \mathrm{~K}$. of $3 \mathrm{~d}$ electrons of a paramagnetic impurity takes place. Hence, some spin polarization of free electrons also occurs due to the interaction with this impurity. The same spin orientation of $3 \mathrm{~d}$ electrons and free electrons results in a decrease of the probability of the spin-flip scattering that is the main mechanism of the momentum relaxation in such interaction. As a consequence, the intensity of the emission that is due to intraband indirect transitions of hot electrons should decrease with applying magnetic field.

The influence of the magnetic field on the intensity of the hot electron emission in the $\mathrm{ZnS}$ :Cr TFELS has been studied at $4.2 \mathrm{~K}$. The results of this investigation are given in Fig. 6. The experimental data (circles) are compared with the dependence of the exchange scattering cross-section $\left(\sigma_{\text {exch }}\right)$ calculated (solid curve) using the relation obtained in [17]:

$\sigma_{\text {exch }}(H) / \sigma_{\text {exch }}(0)=1-P_{e} B_{s} /(s+1)$

where $\quad B_{s}=s \cdot \operatorname{cth}(s \omega / k T)-1 / 2 \operatorname{cth}(\omega / 2 k T) \quad$ is the Brillouin function, $\omega=g \mu_{\mathrm{B}} H, s$ is the paramagnetic center spin and $g$ is its $g$-factor, $\mu_{\mathrm{B}}$ is the Bohr magneton, $k$ is the Boltzmann constant, $T$ is temperature, and $P_{e}=\tanh (\omega / 2 k T)$ is the spin polarization of free electrons.

The experimental dependence $I(H) / I(0)$ on $H$ and the dependence $\sigma_{\text {exch }}(H) / \sigma_{\text {exch }}(0)$ versus $H$ calculated with $s=5 / 2$ and $g=2$ are in the close agreement. These values of $s$ and $g$ are inherent to $\mathrm{Cr}^{+}$ion. Thus, the given experiment confirms the presence of $\mathrm{Cr}^{+}$ions in $\mathrm{ZnS}: \mathrm{Cr}$ films and the exchange scattering on these ions that is one of the channels for momentum relaxation of radiating hot electrons.

\section{Conclusions}

The emission ranged from 0.4 up to $1.0 \mu \mathrm{m}$ in the EL spectrum of the $\mathrm{ZnS}$ :Cr TFELS, which accompanies the more intensive NIR emission of $\mathrm{Cr}^{2+}$ ions, is due to intraband indirect transitions of hot electrons. This is confirmed, on the one hand, by its $I(V)$ dependence identical to the voltage dependence of the NIR emission intensity resulting from the impact excitation of $\mathrm{Cr}^{2+}$ ions by hot electrons and, on the other hand, by a low intensity of this emission and its structureless spectral dependence.

The main mechanism of the momentum relaxation of radiating hot electrons is scattering on charged impurities. This is indicated by the similarity of the experimental dependence of $I$ versus $h v$ and analogous dependence calculated in the assumption of such a scattering mechanism. The steeper decrease of the intensity at $h v>2.3 \mathrm{eV}$ in the experimental dependence than in the calculated one is explained by the photon absorption by the defects arising in $\mathrm{ZnS}$ doped with $\mathrm{Cr}$. Two broad bands with the maxima at $\sim 2.8$ and $\sim 3.5 \mathrm{eV}$, in which the absorption is accompanied by the photocurrent, have been revealed in the PDP spectrum of the $\mathrm{ZnS}: \mathrm{Cr}$ TFELS. It is supposed that the higher-energy

\section{(C) 2005, V. Lashkaryov Institute of Semiconductor Physics, National Academy of Sciences of Ukraine}


band is related to the isovalent trap $\left[\mathrm{Cr}_{\mathrm{Zn}}^{2+}\right]^{0}$ and the second band is due to the photoionization of $\left[\mathrm{Cr}_{\mathrm{Zn}}^{+}\right]^{-}$ acceptor. The latter defect appears owing to the presence of $\mathrm{Cr}^{+}$ions in the $\mathrm{ZnS}: \mathrm{Cr}$ film in addition to the dominant $\mathrm{Cr}^{2+}$ ions. It is known [14] that the $\mathrm{Cr}^{2+} \rightarrow \mathrm{Cr}^{+}$ recharging appears if there are free electrons. Obviously, this recharging results from resonant tunneling of an electron on high excited states of $\mathrm{Cr}^{2+}$ ions. In the $\mathrm{ZnS}: \mathrm{Cr}$ film, the concentration of free electrons is high $\left(>10^{18} \mathrm{~cm}^{-3}\right)$ even at $V<V_{\text {th. }}$. The direct detection of $\mathrm{Cr}^{+}$ ions by the EPR method failed evidently due to their small number in the thin film $(<1 \mu \mathrm{m})$ even when the $\mathrm{Cr}^{+}$ion concentration reaches $\sim 10^{18} \mathrm{~cm}^{-3}$.

The presence of $\mathrm{Cr}^{+}$ions in the $\mathrm{ZnS}: \mathrm{Cr}$ film studied and their participation in the scattering mechanism are confirmed by the magnetic field effect on the intensity of the hot electron emission. It was revealed the decrease of $I$ in the magnetic field at $4.2 \mathrm{~K}$ that depends on $H$ just the same as exchange scattering cross-section calculated in the case of scattering centers with the spin $5 / 2$ and $g$ factor equal to 2. These parameter magnitudes are inherent to $\mathrm{Cr}^{+}$ion with $3 \mathrm{~d}^{5}$ electron configuration.

Thus, the main mechanism of the momentum relaxation of hot electrons during their radiative intraband indirect transitions in the ZnS:Cr TFELS is scattering by $\mathrm{Cr}^{+}$ions. The scattering is caused not only by the Coulomb interaction, but also by the exchange interaction.

Acknowledgement. The authors would like to thank Dr. V. Abramishvili for the participation in the experiment based on using the magnetic field and Dr. B. Shanina for the fruitful discussion of the results.

\section{References}

1. E.M. Conwell, High-field transport in semiconductors, Academic Press, New-York and London, p. 152-216, 360-366 (1967).

2. L.A. Kosyachenko, Scientific Transaction of Tartu State University 655, p. 12 (1983) (in Russian).
3. V.S. Vichnin, M.F. Deigen, Yu.G. Semenov, B.D. Shanina // Phys. Techn. Semicond. 18 (8), p. 2222 (1976) (in Russian).

4. N.E. Rygby, J.W. Allen // J. Phys. C 21, p. 3483 (1988).

5. A.A. Douglas, J.F. Wager, D.C. Morton, J.B. Koh // Appl. Phys. Lett. 63 (2), p. 231 (1993).

6. L.A. Kosyachenko // Izvestiya AN SSSR, Ser. phys. 49 (10), p. 1934 (1985) (in Russian).

7. H.Y. Fan, W. Spitzer, R.J. Collins // Phys. Rev. 101, p. 566 (1956).

8. S. Visvanathan // Ibid. 120, p. 176, 179 (1960).

9. S.M. Ryabchenko, Yu.G. Semenov, in: Spectroscopy of crystals, Nauka, Leningrad, p. 178-196 (1983) (in Russian).

10. A.E. Belyaev, O.P. Gorodnichii, Yu.G. Semenov // Fiz. Tekhn. Poluprov. 22, p. 335 (1988).

11. A.V. Vasilyev, N.A. Vlasenko, Z.L. Denisova, Ya.F. Kononets, A.I. Riskin, A.Ya. Chomyak. In: Optoelectronics and semiconductor technics 25, p. 68 (1993) (in Russian).

12. N.A. Vlasenko, A.I. Beletskii, Z.L. Denisova, Ya.F. Kononets, L.I. Veligura. In: S.V. Svechnikov, M.Ya. Valach (Eds.) Optical diagnosis of materials and devices for opto-, micro-, and quantum electronics // Proc. SPIE 3359, p. 512 (1998).

13. Y.A. Ono, Electroluminescent display, World Scientific, Singapore, p. 7-10 (1985).

14. M. Godlevski, M. Kaminska // J. Phys. C 13, p. 6537 (1980).

15. N.A. Vlasenko, Z.L. Denisova, Ya.F. Kononets, L.I. Veligura, Yu.A. Tsyrkunov, A.A. Vdovenkov. In: Proc. of 12th intern. workshop on inorganic and organic electroluminescence. September 20 - 23, 2004, Toronto, Canada.

16. N.A. Vlasenko, A.I. Beletskii, Z.L. Denisova, Ya.F. Kononets, L.I. Veligura, in: R.H. Mauch and H.E. Gumlich (Eds.), Inorganic and organic electroluminescence, Wissenshaft and Technik Verlag, Berlin, p. 267 (1996).

17. A.E. Belyaev, Ya.F. Kononets, Yu.G. Semenov, N.A. Vlasenko // J. Crystal Growth 101, p. 985 (1990). 